University of Nebraska - Lincoln

DigitalCommons@University of Nebraska - Lincoln

\title{
Centralized vs. Distributed Connection Management Schemes under Different Traffic Patterns in Wavelength-Convertible Optical Networks
}

\author{
Lu Shen \\ University of Nebraska-Lincoln, Ishen@cse.unl.edu \\ Byrav Ramamurthy \\ University of Nebraska-Lincoln, bramamurthy2@unl.edu
}

Follow this and additional works at: https://digitalcommons.unl.edu/cseconfwork

Part of the Computer Sciences Commons

Shen, Lu and Ramamurthy, Byrav, "Centralized vs. Distributed Connection Management Schemes under Different Traffic Patterns in Wavelength-Convertible Optical Networks" (2002). CSE Conference and Workshop Papers. 93.

https://digitalcommons.unl.edu/cseconfwork/93

This Article is brought to you for free and open access by the Computer Science and Engineering, Department of at DigitalCommons@University of Nebraska - Lincoln. It has been accepted for inclusion in CSE Conference and Workshop Papers by an authorized administrator of DigitalCommons@University of Nebraska - Lincoln. 


\title{
Centralized vs. Distributed Connection Management Schemes under Different Traffic Patterns in Wavelength-Convertible Optical Networks
}

\author{
Lu Shen and Byrav Ramamurthy \\ Department of Computer Science and Engineering \\ University of Nebraska - Lincoln \\ Lincoln, NE 68588-0115 USA
}

\begin{abstract}
Centralized and Distributed methods are two connection management schemes in wavelength convertible optical networks. In the earlier work, the centralized scheme is said to have lower network blocking probability than the distributed one. Hence, much of the previous work in connection management has focused on the comparison of different algorithms in only distributed scheme or in only centralized scheme. However, we believe that the network blocking probability of these two connection management schemes depends, to a great extent, on the network traffic patterns and reservation times. Our simulation results reveal that the performance improvement (in terms of blocking probability) of centralized method over distributed method is inversely proportional to the ratio of average connection interarrival time to reservation time. After that ratio increases beyond a threshold, those two connection management schemes yield almost the same blocking probability under the same network load. In this paper, we review the working procedure of distributed and centralized schemes, discuss the tradeoff between them, compare these two methods under different network traffic patterns via simulation and give our conclusion based on the simulation data.
\end{abstract}

Index Terms-Distributed Connection Management, Centralized Connection Management, Wavelength-Convertible Optical Networks, TEL Traffic Pattern, VOD Traffic Pattern, VPN Traffic Pattern, EST Traffic Pattern, Connection Inter-Arrival Time.

\section{INTRODUCTION}

In a WDM optical network, end-users may communicate with one another via all-optical WDM channels, which may span multiple fiber links and are referred to as lightpaths [1]. If a lightpath in a network must occupy the same wavelength across all the traversed fiber links, the network is called wavelength-continuous network. By employing optical switches at intermediate nodes in networks [2], a lightpath may occupy different wavelengths on different links along the route. Such a network is known as a wavelength-convertible network. Connection establishment in WDM networks include a Routing and Wavelength Assignment (RWA) [3] [4]. That is, in order to establish a lightpath, a route from a source node to a destination node and corresponding wavelengths on each fiber link across the route must be decided. The RWA problem under static traffic with the entire set of connections known in advance is known as the Static Lightpath Establishment (SLE) problem. If the network traffic matrix is not known in advance, the RWA problem becomes the Dynamic Lightpath Establishment (DLE) problem. The SLE problem with wavelength-continuity constraint can be formulated as an integer linear program, which is

This work was supported in part by NSF grants (ANI-0074121 and EPS0091900).
NP-complete. Its objective is to minimize the number of wavelengths needed to setup a certain set of lightpaths for a given physical topology. For DLE, it is to maximize the number of connections that need to be established in the network at any time. Normally, RWA can be divided into two independent subproblems, routing and wavelength assignment. The work in [5] compared different routing approaches, such as fixed, fixedalternate and adaptive routing. The paper also reviewed most of wavelength assignment algorithms proposed in the literature, such as random wavelength assignment, First-Fit, Lease-Used, Most-Used, Min-Product, Least Loaded, Max-SUM, Relative Capacity Loss and so on. Due to the complexity of combining routing and wavelength assignment to achieve good network performance, it is still an active field of research.

RWA solves the wavelength assignment and routing problems in networks. The Connection management mechanism is concerned with how to make use of RWA to setup and release connections. Different connection management mechanisms work under different working procedures, which may use the same RWA algorithm. For example, both distributed and centralized connection management methods in wavelengthconvertible networks may use the fixed shortest hop path routing and First-Fit wavelength assignment algorithm.

Substantial effort has been made in comparing different connection management mechanisms for different networks. In [7] and [8], in terms of link utilization and network throughput, the network performance of different distributed connection management schemes, such as destination initiated vs. source initiated reservation, one-way vs. two-way reservation, spend-thrift vs. frugal reservation etc., are compared by simulation in both wavelength-continuous and wavelength-convertible networks. In [9], the comparison of centralized and distributed reservation mechanisms in WDM ring can be found.

Most of the above work, except for the work in [9], concentrates on comparison of distributed connection management schemes in wavelength-continuous networks under a fixed traffic pattern. We believe the effectiveness of fast circuit switching depends on both the traffic characteristics and reservation mechanism. A comparison of distributed and centralized reservation mechanisms under different traffic patterns in wavelength-convertible mesh network is not available in the literature and will be the focus of this paper.

The rest of this paper is organized as follows. In Section II, we review the working procedure of centralized and distributed connection management, followed by an introduction of race 
condition in the distributed scheme. In Section III, we describe our simulation and analyze the network performance of these two schemes under different traffic patterns. In Section IV, we conclude the paper.

\section{Centralized And Distributed Connection MANAGEMENT SCHEMES}

\section{A. Working procedure}

For centralized connection management scheme, a central controller or central network management system holds the global information of network resources, such as network topology, link states, wavelength usage on each link and the states of each network element. When a source node needs to transfer data, a connection request is sent to the central controller and then a route and corresponding wavelengths are calculated, according to the routing and wavelength assignment algorithm at the controller, based on the global information of network resources. Then, the controller reserves and configures resources for the connection by notifying each node on the route. Notification message can be sent out either in a hop-byhop or a parallel manner depending on the detailed implementation. If the controller finds there are not sufficient network resources to support a connection, the connection will be blocked. After the controller receives acknowledgment from each node, it will send a message, notifying the source node to send data on reserved wavelengths along the route. When a connection is finished, the controller will signal each node involved to release the selected wavelengths.

For the distributed approach, each node on the route will be involved in making decision on selecting the wavelengths. The connection request goes through each node along the route and reserves wavelengths based on the local information at the node. After the connection request arrives at the destination node, an ACK message is sent back on the reverse path to configure the reserved wavelengths at each node. When the source node receives this ACK, it will start sending data using the reserved wavelengths on the route. If one of the intermediate nodes on the reserved route does not have the available wavelength for this connection, the connection request will be blocked and then an NACK will be sent back to the source node to release the previous reserved wavelengths. When a connection is torn down, a release request will be sent to the destination to release the network resources being used at each node. Lightpath establishment is decided by coordination among different nodes, not only by a central controller. It is called a distributed approach due to its distributed nature of operation.

The Centralized method is vulnerable due to its single point of failure and lacks of scalability and interoperability. Furthermore, the central controller is a computation bottleneck for large-scale networks. But it is simpler to implement and more suitable for small-sized networks.

Compared to the centralized approach, the distributed one is more suitable for large-scale networks. It leaves the decision of selecting a local wavelength and the next-hop to each node and distributes the computation task to each node along the route. So, it eliminates the bottleneck from the central controller and improves the reliability of networks. Under the dis-

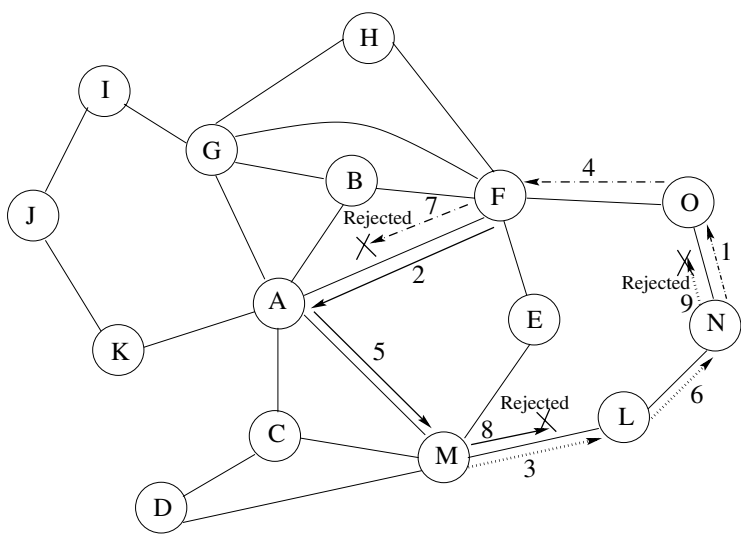

Fig. 1. An example of race condition in the distributed connection management scheme. (Explanation is in Section II-B)

tributed scheme, different vendors' network elements can communicate with one another using well-known routing and signaling protocols. Therefore, it provides better scalability and interoperability by increasing the complexity of the system.

Considering the working styles of these two methods, the distributed scheme has a higher blocking probability than the centralized one, due to its distributed working fashion. Nevertheless, from our simulation, we find that the network traffic pattern and reservation time can affect the blocking probability to a great extent that under different traffic patterns with the same network load, these two schemes may have the same blocking probability.

\section{B. Race condition}

One of the reasons why most people believe that the distributed scheme has a higher blocking probability is that its distributed working style may introduce a race condition, which does not exist in centralized scheme. In the distributed scheme, if requests for several calls enter the network in a very close interval, each of them may reserve resources necessary for another; If this hold-and-request forms a loop, a race condition occurs and all these call requests are blocked even if the network may have resources for some of them. The centralized scheme uses a first-come-first-served (FCFS) method to allocate resources based on the global information, which can ensure that call blocking due to race condition is impossible. Race condition is clearly shown by the example in Figure 1.

The network, in Figure 1, is working under the distributed connection management scheme. Now we consider the situation that node $\mathrm{N}$ wants to send data to node $\mathrm{K}$ (dash-dotted line), node $\mathrm{F}$ wants to send data to node $\mathrm{L}$ (solid line), and node $\mathrm{M}$ wants to send data to node $\mathrm{O}$ (dotted line). We assume the reservation route is computed, according to shortest hop path, as follows: N-O-F-A-K, F-A-M-L and M-L-N-O . And, currently only one wavelength is available in each fiber link. The events in Figure 1 happen in the following orders: (Steps 1, 2 and 3 happen at the same time. Steps 4, 5 and 6 happen at the same time and Steps 7, 8 and 9 happen at the same time.)

1) Node $\mathrm{N}$ sends a connection request to node $\mathrm{O}$ and reserves one wavelength on the link N-O. 


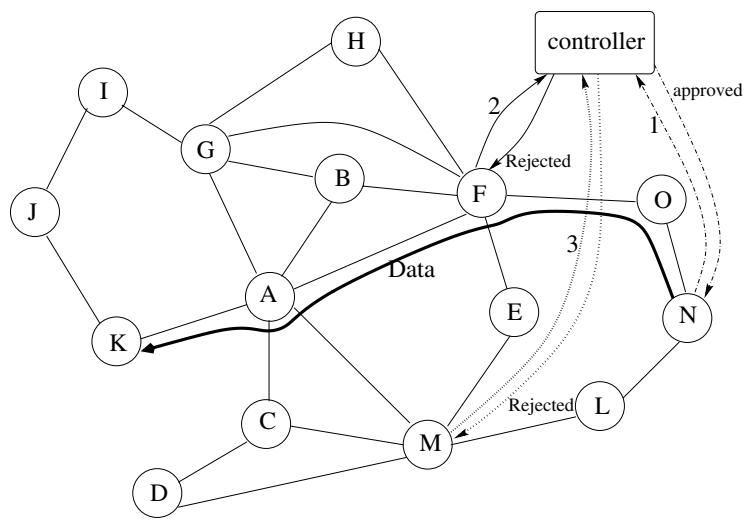

Fig. 2. Pacific Bell mesh network with a centralized controller.

2) Node $F$ sends a connection request to node $A$ and reserves one wavelength on the link F-A.

3) Node $M$ sends a connection request to node $L$ and reserves one wavelength on the link M-L. (Note: now there is no wavelength available on the paths N-O, F-A and ML )

4) Node $O$ sends a connection request to node $F$ and reserve one wavelength on the link O-F.

5) Node $A$ sends a connection request to node $M$ and reserve one wavelength on the link A-M.

6) Node $\mathrm{L}$ sends a connection request to node $\mathrm{N}$ and reserve one wavelength on the link L-N.

7) Node $F$ attempts to send a connection request to node $A$ and the request is rejected due to lack of wavelength on the link F-A.

8) Node $\mathrm{M}$ attempts to send a connection request to node $\mathrm{L}$ and the request is rejected due to lack of wavelength on the link M-L.

9) Node $\mathrm{N}$ attempts to send a connection request to node $\mathrm{O}$ and the request is rejected due to lack of wavelength on the link N-O.

As a result, all the three connection requests are rejected, although there are enough network resources, i.e. wavelengths, for one of the above connection requests. If the centralized connection method is used, this problem is tackled automatically due to the FCFS rule at the central controller. As shown in Figure 2, the connection request from node $\mathrm{N}$ is received and processed first at the central controller. So, node $\mathrm{N}$ obtains the network resources for sending data along the path N-O-F-A-K. The other two requests, originated from nodes $\mathrm{F}$ and $\mathrm{M}$, are rejected due to lack of wavelengths.

From the above example, we conclude that the distributed connection scheme has the potential to have a higher blocking probability due to race conditions. However, if connection requests arrive in an infrequent manner, such that only one connection request exists in the network at any time, distributed method may yield the same blocking probability as the centralized one. This condition is satisfied when the ratio of average connection inter-arrival time to reservation time is large.

In Section II-A, the discussion of the distributed approach assumes that each node lacks of global knowledge of network
TABLE I

CONSTANT PARAMETERS USED IN THE EXPERIMENTS.

\begin{tabular}{|c|c|}
\hline Parameter & Value \\
\hline \hline Network Topology & Pacific Bell \\
\hline Number of Calls & 100000 \\
\hline Number of Wavelengths per Link & 8 \\
\hline Switching Time & $10 \mathrm{~ms}$ \\
\hline Transmission Time & $0.5 \mathrm{~ms}$ \\
\hline Propagation Delay & $\begin{array}{c}\text { Proportional to } \\
\text { lightpath length }\end{array}$ \\
\hline Wavelength Assignment & FIRST FIT \\
\hline Routing Algorithm & Static Shortest Hop Path \\
\hline Source-destination Distribution & Uniform distribution \\
\hline Inter-arrival Time Distribution & Exponential distribution \\
\hline Service Time Distribution & Exponential distribution \\
\hline
\end{tabular}

resources. Link state advertisement (LSA) can be used to flood network state information and ensure every node keep the same picture of the whole network. By this method, constraint-based routing can be implemented at the source node. However, this cannot eliminate race conditions, because multiple nodes may be computing lightpaths simultaneously. One of the methods to avoid race conditions is to retry the connection after a block happens. This is beyond the scope of this paper and may be examined in the future.

\section{EXPERIMENT}

We conducted a series of discrete event simulations using the SIMON simulator ([10]) to study the effects of various network parameters on network blocking probability for distributed and centralized connection management methods. In this section, we first present the assumption of the simulation condition. Then, we analyze simulation results and study the impact of different traffic patterns on the blocking probability for distributed and centralized connection schemes.

\section{A. Simulation}

Blocking probability is used as the measure of network performance in this paper. It is defined as the ratio of the number of blocked calls to the number of offered calls (also referred to as connection requests). Through observing the variation of blocking probability with different network load, service time and processing time, we make a conclusion on how traffic patterns and reservation times affect the blocking probability. In Table 1, we show those parameters which are kept constant all the time in the simulation. In Table 2, all of the variable parameters are listed.

The simulation is conducted on the Pacific Bell network topology, the same network topology shown in Figure 1 and Figure 2, which has 15 nodes, 21 bi-directional 100-km links and 8 wavelengths on each unidirectional link. The switching time, the time for configuring the switch at each node, is fixed at $10 \mathrm{~ms}$ for each node. The transmission time, the duration for sending a reservation message, is fixed at $0.5 \mathrm{~ms}$. The propagation delay is proportional to the corresponding lightpath length. 
TABLE II

VARIABLE PARAMETERS USED IN THE EXPERIMENTS.

\begin{tabular}{|c|c|}
\hline Parameter & Value \\
\hline \hline Connection Management Scheme & $\begin{array}{c}\text { Distributed vs. } \\
\text { Centralized }\end{array}$ \\
\hline Network Load (Erlang) & $20,30,40,50$ \\
\hline Processing Time (ms) & $1,10,50,100$, \\
& 1000,2000 \\
\hline Service Time (s) & 30,300, \\
& 3000,3000000 \\
\hline
\end{tabular}

We choose First-Fit algorithm and static shortest hop path as RWA. We assume a uniform distribution of source-destination pairs and an exponential distribution of both inter-arrival time and service time. 100,000 calls are offered, which are uniformly distributed over all source-destination pairs. For each source-destination pair, calls arrive following a Poisson distribution.

The network load is defined as the ratio of the connection arrival rate to the connection service rate, i.e. the ratio of average service time to average inter-arrival time:

$$
\text { network load }=\frac{\text { average service time }}{\text { average inter-arrival time }}
$$

The inter-arrival time is the interval between two calls and the service time is the duration for serving a connection. From equation (1), we know that under the same network load, the average inter-arrival time is proportional to the average service time. In other words, under the same service time, inter-arrival time is inversely proportional to the network load.

The simulation takes into consideration the variation of processing time, network load and traffic pattern (in terms of the service time or the inter-arrival time). We define four different traffic patterns as following:

- EST - the extremely short traffic pattern with average service time of 30 seconds.

- TEL - the telephony-like traffic pattern with average service time of 300 seconds ( 5 minutes).

- VOD - the video on demand traffic pattern with average service time of 3,000 seconds (50 minutes).

- VPN - the virtual private network traffic pattern via lease contract with average service time of 300,000 seconds (3 days and 11 hours and 20 minutes).

In the simulation, we assume that the network has full wavelength conversion capability and out-of-band signaling is used. Under each traffic pattern, we conduct our simulation with different processing times and different network loads (different inter-arrival times). Then, we obtain a series of blocking probability values under different traffic patterns, network loads and processing times. The simulation is conducted under distributed and centralized schemes respectively and the final data is plotted in Figure 3, Figure 4, Figure 5, and Figure 6. Each line in those figures presents the values of the blocking probability for different processing times.

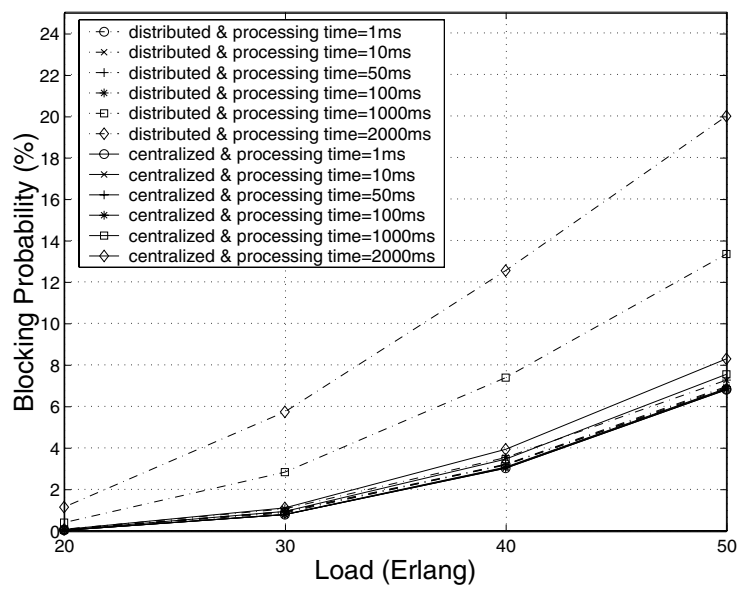

Fig. 3. Blocking probability vs. network load under EST traffic pattern

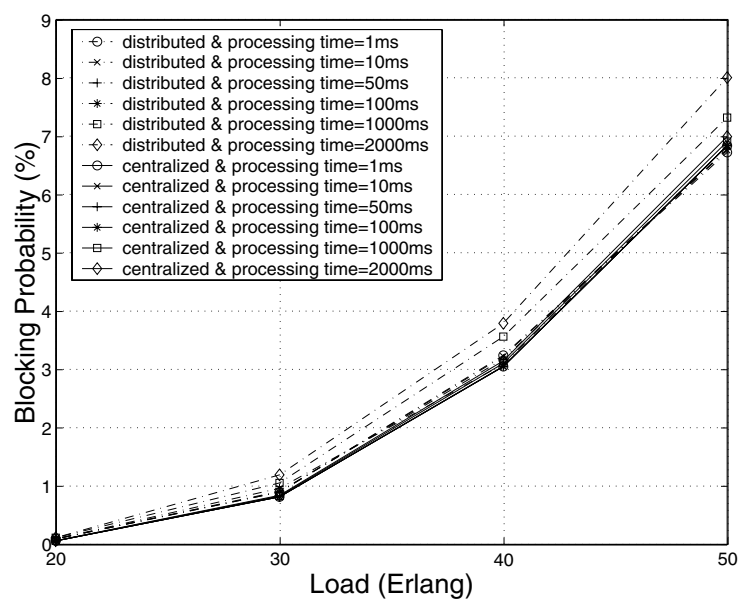

Fig. 4. Blocking probability vs. network load under TEL traffic pattern

\section{B. Data Analysis}

The reservation time is the sum of transmission time, processing time, switching time (at each node) and propagation delay. Since all the above parameters but processing time are constant in our simulation, the change in processing time reflects the change of reservation time.

First, we compare the blocking probability of the centralized scheme and the distributed scheme under different traffic patterns. From the earlier work [6], we expect the centralized connection management method have a lower blocking probability than the distributed scheme. However, this effect is only obvious under TEL, when processing time is $1000 \mathrm{~ms}$ and $2000 \mathrm{~ms}$, and under EST, when processing time is $100 \mathrm{~ms}, 1000 \mathrm{~ms}$ and $2000 \mathrm{~ms}$. Under the other traffic patterns and for those with lower processing time under TEL and EST, the distributed and centralized schemes almost result in the same blocking probability for the same load. Because the distributed scheme may introduce race conditions, its blocking probability could be higher than that of the centralized scheme. However, if the connection requests arrive infrequently such that each connection request arrives after the reservation of other requests are already done, the blocking probability of the distributed and centralized schemes should be the same. In other words, if the ratio 


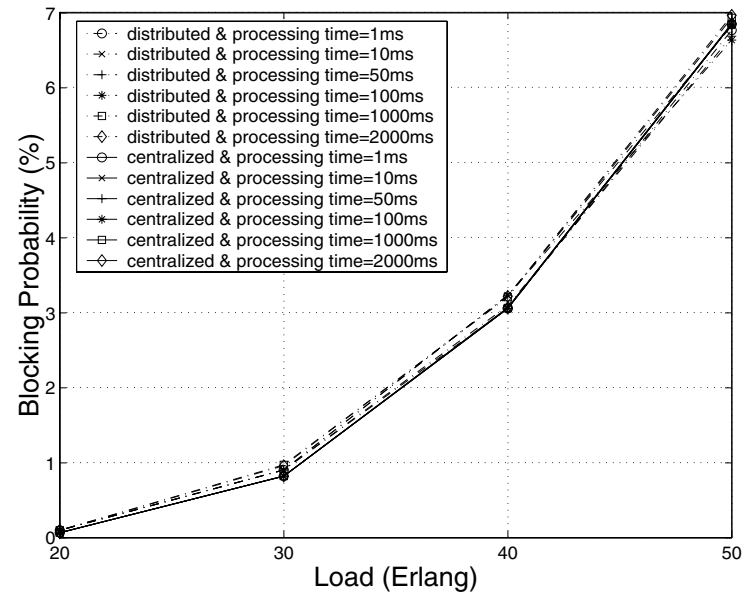

Fig. 5. Blocking probability vs. network load under $V O D$ traffic pattern

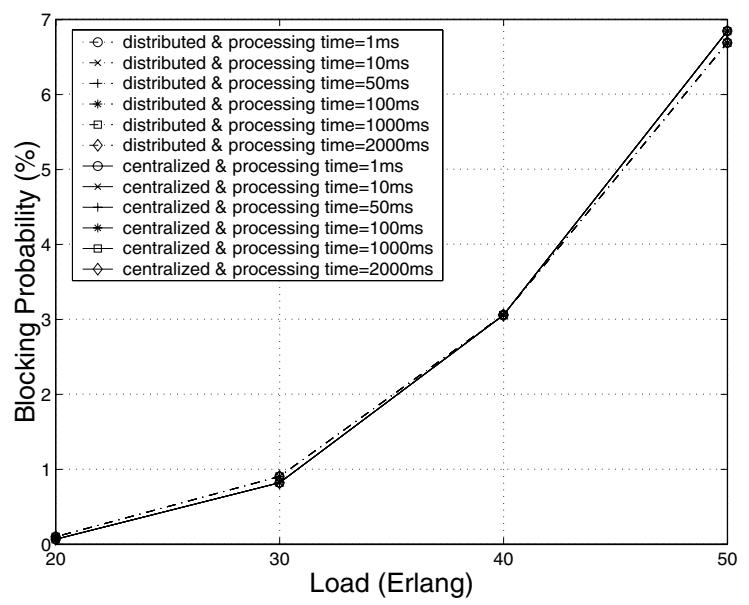

Fig. 6. Blocking probability vs. network load under $V P N$ traffic pattern

of the average inter-arrival time to the processing time is large enough, there exists no difference in the blocking probability between the distributed and centralized schemes. Simulation results show that when the service time or inter-arrival time increases beyond 100 times of the processing time, the performance improvement of the centralized scheme over distributed scheme is negligible.

Second, we notice that the processing time of the centralized scheme has very little impact on the blocking probability under all kinds of traffic patterns. Since the controller has a global knowledge of the network resources and the requests in the queue at the central controller are processed according to FCFS rule, race conditions will not happen in a centralized scheme. So, connection requests will not compete with each other for wavelengths as illustrated in the Figure 1. For the distributed scheme, blocking probability grows as the processing time increases, when the ratio of the average inter-arrival time to the processing time is more than 100 . The reason for this phenomenon is that as the processing time increases, the probability of having a race condition also increases. As a result, more connection requests are blocked.

Third, as the network load grows, the blocking probability increases dramatically for both centralized and distributed schemes. Also, the performance improvement of the centralized scheme over the distributed scheme increases. For the same service time, known from equation (1), the value of interarrival time decreases as the network load increases. This causes more intensive competition on network resources.

All of the above observations reveal that the network performance improvement, in terms of blocking probability, of the centralized scheme over the distributed scheme depends greatly on the network traffic patterns and reservation times.

\section{CONCLUSION}

This paper compares the centralized and distributed connection management schemes under different traffic patterns in wavelength-convertible WDM networks. The simulation results reveal that, in terms of network blocking probability, the network performance improvement of the centralized connection scheme over the distributed scheme is inversely proportional to the ratio of the average connection inter-arrival time to reservation time. After the ratio of the average connection inter-arrival time to reservation time increases beyond a threshold, the distributed and centralized mechanisms yield the same blocking probability under the same network load.

Besides network blocking probability, the decision on choosing a connection management scheme also depends on other factors, such as scalability, interoperability, reliability, cost and so on. Recent research shows that the distributed connection management scheme is preferred in wavelength-convertible WDM networks, because its distributed working style fits with the recently proposed Multiple-Protocol Lambda Switching (MPLambdaS [11]) technology.

\section{REFERENCES}

[1] B. Mukherjee, Optical Communication Networks, McGraw-Hill, New York, 1997.

[2] B. Ramamurthy and B. Mukherjee, "Wavelength Conversion in WDM Networking," IEEE Journal on Selected Areas in Communications, vol. 5, pp. 691-703, Sept. 1998.

[3] R. Ramaswami and K.N. Sivarajan, "Routing and Wavelength Assignment in All-Optical Networks," IEEE/ACM Transactions on Networking, vol. 3, no. 5, pp. 489-500, Oct. 1995.

[4] D. Banerjee and B. Mukherjee, "A Practical Approach for Routing and Wavelength Assignment in Large Wavelength-Routed Optical Networks," IEEE Journal on Selected Areas in Communications vol 14, no. 5, pp. 903-908, June 1996.

[5] H.Zhang, J. Jue and B. Mukherjee, "A Review of Routing and Wavelength Assignment Approaches for Wavelength Routed Optical WDM Networks," Optical Networks, Vol, No. 1, Jan 2000.

[6] Byrav Ramamurthy, Lu Shen and Elie Sawma, "Connection Management for Wavelength-routed Optical WDM Networks," book chapter in "Advances in Optical Networks", Kluwer Academic Publishers, 2001.

[7] Y. Mei and C. Qiao, "Distributed Control Schemes for Dynamic Lightpath Establishment in WDM Optical Networks," Optical Network Workshop, Richardson, TX, Jan 1999.

[8] Y. Mei and C. Qiao, "Efficient distributed control protocols for WDM optical networks," Proc. Int'l Conference on Computer Communication and Networks, pages 150-153, September 1997.

[9] J. Cai, A. Fumagalli, Ch. Guan, "Centralized vs. Distributed On-Demand Bandwidth Reservation Mechanisms in WDM Ring," Optical Fiber Conference, Anaheim, CA, 2001.

[10] B. Ramamurthy, D. Datta, H. Feng, J.P. Heritage, and B. Mukherjee, "SIMON: A Simulator for Optical Networks", All Optical Networking, 1999: Architecture, Control and Management Issues (J.M. Senior, C. Qiao, and S.Dixit, eds.), vol 3843, pp. 130-135, Proceeding of SPIE, Sep. 1999.

[11] N. Ghani, "Lambda Labeling: a Framework for IP-Over-WDM using MPLS," Optical Networks Magazine, vol 1, no 2, April 2000. 